\title{
Does emotional reasoning change during cognitive behavioural therapy for
}

\author{
anxiety?
}

Short title: Does emotional reasoning change with CBT?

Word count: 5407 (including references)

David Berle ${ }^{1,2}$, Michelle Moulds ${ }^{1}$, Vladan Starcevic ${ }^{3}$, Denise Milicevic ${ }^{2}$, Anthony Hannan², Erin Dale ${ }^{2}$, Kirupamani Viswasam ${ }^{4} \&$ Vlasios Brakoulias ${ }^{3}$.

1. School of Psychology, UNSW, Australia.

2. Nepean Anxiety Disorders Clinic, Nepean Blue Mountains Local Health District, Australia.

3. Discipline of Psychiatry, Sydney Medical School - Nepean, University of Sydney, Australia.

4. Department of Psychiatry, Nepean Hospital, Penrith, Australia.

Address for correspondence:

David Berle

School of Psychiatry, UNSW Australia

Black Dog Institute Building

Hospital Road, Prince of Wales Hospital,

Randwick NSW 2031

Australia

Email: d.berle@unsw.edu.au

Ph: +61293828503

Fax: +6129382 8151

Acknowledgements:

Karen Moses is thanked for her assistance with participant recruitment. 


\begin{abstract}
Emotional reasoning refers to the use of subjective emotions, rather than objective evidence, to form conclusions about oneself and the world (Arntz, Rauner, \& van den Hout, 1995). It is a key interpretative bias in cognitive models of anxiety disorders and appears to be especially evident in individuals with anxiety disorders. However, the amenability of emotional reasoning to change during treatment has not yet been investigated. We sought to determine whether emotional reasoning tendencies change during a course of routine cognitivebehavioural therapy (CBT). Emotional reasoning tendencies were assessed in 36 individuals with a primary anxiety disorder who were seeking treatment at an outpatient clinic. Changes in anxiety and depressive symptoms as well as emotional reasoning tendencies after 12 sessions of CBT were examined in 25 individuals for whom there was complete data. Emotional reasoning tendencies were evident at pretreatment assessment. Although anxiety and depressive symptoms decreased during CBT, only one of six emotional reasoning interpretative styles (pertaining to conclusions that one is incompetent) changed significantly during the course of therapy. Attrition rates were high and there was not enough information regarding the extent to which therapy specifically focused on addressing emotional reasoning tendencies. Individuals seeking treatment for anxiety disorders appear to engage in emotional reasoning, however routine individual CBT does not appear to result in changes in emotional reasoning tendencies.
\end{abstract}

Keywords:

Emotional reasoning; cognitive bias; anxiety; CBT; treatment outcome; cognitive distortion. 
Emotional reasoning refers to a reliance on one's emotional state at the expense of objective information when forming conclusions about oneself and the world (Arntz, Rauner, \& van den Hout, 1995). Beck and colleagues identified emotional reasoning (also referred to as "mistaking feelings for facts”) as a key cognitive distortion that was considered to contribute to the development and maintenance of emotional disorders. In the context of anxiety, emotional reasoning has been described as a process whereby an individual may reason that "If there is danger, I feel anxious" and in turn assume that "If I feel anxious, there must be danger” (Arntz et al., 1995).

Emotional reasoning appears to characterise anxiety disorders. Arntz et al. (1995) compared emotional reasoning tendencies in treatment-seeking individuals with panic disorder, spider phobia, social anxiety and a mixed anxiety disorders group with a nonclinical control group. In contrast with the control group, each of the anxiety disorder groups demonstrated elevated levels of emotional reasoning. Interestingly, emotional reasoning was not confined to situations relevant to one's own disorder. For instance, individuals with panic disorder appeared to engage in emotional reasoning when imagining social anxiety themed scenarios. Emotional reasoning also appears to characterise posttraumatic stress disorder (Engelhard, Macklin, McNally, van den Hout, \& Arntz, 2001), although it remains unclear to what extent dysphoria and depression are characterised by this process (Berle \& Moulds, 2013a, 2013b).

The extant literature then, provides some support that anxiety disorders are characterised by emotional reasoning, consistent with the assertions of cognitive models of emotional disorders (Beck \& Emery, 1985). However, a crucial question pertains to the relationship between emotional reasoning and anxiety symptoms across time. If emotional reasoning contributes to the perpetuation of anxious affect, as cognitive theories of anxiety 
disorders would assert, then emotional reasoning tendencies would be expected to decrease in concert with reductions of anxiety symptoms during treatment of anxiety disorders. Unlike the extensive literature documenting therapy-related changes in other cognitive distortions described by CBT models - such as catastrophizing (Hicks et al. 2005) - changes in emotional reasoning have not yet systematically assessed in routine face-to-face therapy.

The present study aimed to investigate emotional reasoning in a sample of adults receiving routine treatment at a suburban clinic for the treatment of anxiety disorders. The two key questions were: 1 ). whether a sample of treatment-seeking individuals would demonstrate emotional reasoning, and 2). whether emotional reasoning tendencies would change during the course of routine cognitive-behavioural therapy (CBT) for anxiety disorders. So far as the first aim is concerned, it was hypothesised that, consistent with the findings of Arntz et al. (1995), individuals seeking treatment for an anxiety disorder would demonstrate emotional reasoning. With regard to the second aim of the study, it was hypothesised that emotional reasoning tendencies would change in tandem with anxiety symptoms during the course of routine CBT, even if there were no specific interventions included in the treatment to specifically address these tendencies. It was expected that the skills that are conveyed to clients during standard CBT, such as seeking objective evidence for one's assumptions and interpretations, would also be beneficial in reducing unhelpful emotional reasoning tendencies (where the “evidence” may not support an emotionally-laden inference).

Investigating these questions in a routine-therapy context necessarily lacks some of the controls that increase the internal validity of controlled trials that are conducted in research settings, such as manualised treatment, treatment fidelity checks, and prioritisation of participant recruitment and retention. However, on the other hand, recruitment through a routine community-based treatment setting allows improved external validity: it allows 
generalization of findings to the ways in which CBT is administered by practitioners in "realworld” settings, where service demands and clinician resources can result in unavoidable deviations from empirically-based treatment protocols in order to tailor therapy to each individual client's presentation (Verwoerd et al., 2013). The present study was conducted in a routine treatment setting in the knowledge that the findings would reflect relationships between these variables as they are reported by treatment seeking individuals in a routine community treatment environment.

\section{Method}

\section{Participants}

The sample comprised 36 individuals seeking treatment at a community-based outpatient anxiety disorders clinic (see Figure 1).

All participants provided informed consent to participate in the study and ethics committee approval was obtained from the Local Health District Human Research Ethics Committee. Participants were reimbursed at the rate of AUD\$20 per hour for their time.

\section{Routine cognitive-behavioural therapy (CBT)}

Participants received routine individual CBT from one of four experienced clinical psychologists at the clinic (minimum years of post-qualification experience $=5$, maximum $=$ 13) and were assessed at the start of therapy (typically following one or two standard therapy assessment sessions, but before active therapy commenced) and after 12 sessions of therapy, or following their final therapy session (whichever came first) ${ }^{1}$.

The therapy interventions were not standardised, but rather, each clinician tailored the content of therapy according to the particular presenting problems of each participant. In this respect, the therapy reflected routine clinical practice at the clinic. Nonetheless, routine peer

\footnotetext{
${ }^{1}$ We chose to conduct the "posttreatment" assessment after a maximum of 12 sessions as this corresponds to the number of sessions included in numerous CBT protocols. It also ensured some degree of consistency regarding the "dose" of CBT.
} 
consultation between each of the therapists over the course of a number of years (over 10 years in the case of three of the therapists) has helped to ensure a degree of consistency in the way in which cases are formulated and treated. Additionally, a record was kept of the focus of each therapy session to provide a general overview of the nature of therapy that participants received.

Measures

Semi-structured diagnostic interview

All participants were administered the Mini Neuropsychiatric Interview (MINI; Sheehan et al., 1998) by the same researcher (DB) to determine primary and co-occurring DSM-IV diagnoses (American Psychiatric Association, 2013). Thirteen interviews were recorded and co-rated by an independent rater (a clinical psychologist of 10 years experience $)^{2}$. There was $100 \%$ agreement between raters for diagnoses of panic disorder, generalised anxiety disorder (GAD) and social anxiety disorder. There were two disagreements across the 13 interviews rated for obsessive-compulsive disorder and major depression (i.e., 84.6\% agreement; Cohen's Kappa = 0.68).

\section{Self-report questionnaires}

The following self-report questionnaires were administered:

The Beck Anxiety Inventory (BAI; Beck, Epstein, Brown, \& Steer, 1988) is a 21-item self-report scale that assesses common features of anxiety, such as nervousness, a fear of losing control, and somatic aspects of anxiety. Each item is rated on a four-point scale ranging from 0 (not at all) to 3 (severely) and a total score is calculated by summing the item scores. The BAI has shown satisfactory test-retest reliability over 5-weeks in panic disorder and agoraphobia patients ( $r=0.83$; de Beurs, Wilson, Chambless, Goldstein, \& Feske, 1997)

\footnotetext{
${ }^{2}$ The interrater analysis for the diagnosis of GAD was based on 12 rather than 13 co-ratings due to a fault with one of the recordings.
} 
and it has good concurrent validity with other anxiety scales (e.g., $r=0.81$ with the SCL-90R [Derogatis, 1983] anxiety subscale; Steer, Ranieri, Beck, \& Clark, 1993). In the present sample, the internal consistency (Cronbach's $\alpha$ ) was 0.96 .

The Beck Depression Inventory II (BDI-II; Beck, Steer, \& Brown, 1996) is a 21-item self-report scale that assesses symptoms of depression. Each item is rated on a four-point scale ranging from 0 (not at all) to 3 (severely) and a total score is obtained by summing the item scores. Patients diagnosed with major depression have been found to score higher on the BDI-II than those without depression (Arnau, Meagher, Norris, \& Bramson, 2001) and the BDI-II correlates more strongly with other measures of depression than with measures of anxiety (Beck et al., 1996). In the present sample, the internal consistency of the BDI-II was 0.93.

The Beliefs about Emotions Questionnaire (BEQ), Cognitive Emotion Regulation Questionnaire (CERQ) and Spontaneous Use of Imagery Scale (SUIS) were also administered, however the results for these are not reported here as they were included for the purpose of assessing thematically unrelated research questions that will be reported elsewhere.

\section{Experimental emotional reasoning task}

Participants were also administered an emotional reasoning experimental procedure. The procedure was based on the scenario-based procedure of that of Arntz et al. (1995) which has been used in numerous studies of both children (e.g., Muris, Merkelbach, \& van Spauwen, 2003) and adults (e.g., Engelhard et al., 2001).

For the purpose of this study, we used four anxiety-themed scenarios. Two of the scenarios pertained to panic attacks and agoraphobia (feeling a pain in one's chest on the way to the local shopping centre and feeling breathless on a crowded train); one to generalised anxiety disorder (receiving a late bill and worrying about how to pay it); and one to health 
anxiety (noticing a spot on one's skin and wondering if it's cancer). In developing the scenarios, we gave a relatively greater focus to panic disorder than to other anxiety disorders, as panic disorder with or without agoraphobia has historically comprised about half of the overall diagnostic mix for individuals attending the Nepean Anxiety Disorders Clinic. Participants were asked to vividly imagine themselves in the situation described by each of the scenarios. Each of the four scenarios were presented four times during each assessment appointment with a different ending each time: (1). objectively neutral and with a nonvalenced emotional response, (2). objectively neutral with an anxious response, (3). objectively negative ending and a non-valenced emotional response, and (4). objectively negative ending with an anxious response. For instance, the four variations of the first scenario were as follows:

1a. As you walk towards your local shopping centre to buy some groceries, you start to notice that you feel hot and a little bit weak. You are not bothered by this and you remind yourself that it’s a very hot day. (neutral situation and non-anxious emotional response).

1b. As you walk towards your local shopping centre to buy some groceries, you start to notice that you feel hot and a little bit weak. You start to feel anxious and fearful. (neutral situation and anxious emotional response).

1c. As you walk towards your local shopping centre to buy some groceries, you start to feel a crushing pain in your chest and tingling in your arms. You're not bothered by this and remind yourself that it may just be a "stitch" or some momentary passing sensations. (objectively threatening situation and non-anxious emotional response).

1d. As you walk towards your local shopping centre to buy some groceries, you start to feel a crushing pain in your chest and tingling in your arms. You feel terrified about what this might mean. (objectively threatening situation and anxious emotional response).

Each scenario and the respective endings are included in Supplementary file 1. 
Participants were asked to provide the following ratings for each of the four scenarios (i.e., six ratings for each of four endings of each of four scenarios $=96$ ratings in total):

1. How dangerous is this?

2. How negative is this?

3. How likely is it that something bad will occur?

4. How likely is it that you would be unable to cope?

5. How incompetent does this situation suggest that you are?

6. How bad is the worst possible outcome in this situation?

The first rating of perceived danger was used for consistency with the study of Arntz et al. (1995; where ratings of danger were the primary anxiety dependent variable). Ratings 2 and 3 were intended to capture estimates of perceived likelihood of unfavourable outcomes and rating 6 was included to capture the perceived cost of the unfavourable event, consistent with notions that threat appraisals are driven by perceived probability and cost estimates (Uren, Szabó, \& Lovibond, 2004). Ratings 2, 4 and 5 were included as they appeared relevant on the basis of clinical experience. Consistent with previous researchers who have used the scenarios-based emotional reasoning procedure (Berle \& Moulds, 2013a, 2013b; Engelhard et al., 2001), we defined emotional reasoning as the difference between the respective ratings of scenarios with and without anxiety-response information included. In this respect, emotional reasoning scores were not so much a reflection of the content of the scenarios themselves, but rather, an index of the extent to which anxiety-related emotions are associated with negatively-toned interpretations.

\section{Posttreatment assessment}

At the posttreatment assessment, all self-report questionnaire measures were readministered as well as the experimental emotional reasoning procedure. The posttreatment emotional reasoning items were the same as those administered at the pretreatment 
assessment. Previous research indicates that scores on the scenarios-based emotional reasoning procedure have adequate test-retest reliability (test-retest score correlations of 0.75 across 2-6 weeks in Arntz et al. 1995), thus providing confidence that changes in scores on the task may reflect actual changes in emotional reasoning as opposed to measurement error.

\section{Data analyses}

All analyses were conducted in SPSS version 22. Chi-square and independent samples t-tests were used to compare the demographic and clinical characteristics of participants who did and did not complete therapy

To determine whether emotional reasoning scores changed between pre and posttreatment, we used repeated measures $t$-tests of the calculated emotional reasoning difference scores.

\section{Results}

Table 1 summarises the sample characteristics at the pretreatment assessment.

Thirty-three participants (91.7\%) had at least one co-occurring diagnosis at the pretreatment assessment ${ }^{3}$. The numbers and proportions of participants with co-occurring diagnoses is outlined in Table 2.

\section{Emotional reasoning tendencies at pretreatment}

The first hypothesis of the study was that individuals seeking treatment for an anxiety disorder would demonstrate emotional reasoning tendencies. Consistent with this hypothesis, all emotional reasoning difference scores were significantly greater than zero (all $p s<0.05$ and significant after correction for multiple comparisons), suggesting that participants rated situations as more dangerous/negative when an anxious emotional response was indicated.

\footnotetext{
${ }^{3}$ For the purpose of the present study, a participant's "primary" disorder was the condition that the individual was seeking help for and considered to be the most interfering in their life currently.
} 
The means $(M)$, $t$-values, $p$-values and Cohen's $d$ effect-sizes for the ratings were as follows (all $d f=35)$ : how dangerous the situation is $(M=16.85, t=7.05, p<0.0001, d=1.18)$; how negative the situation is ( $M=19.28, t=8.36, p<0.0001, d=1.39)$; how likely that something bad will occur $(M=16.86, t=6.86, p<0.0001, d=1.14)$; how likely that unable to cope ( $M=16.37, t=7.64, p<0.0001, d=1.27)$; how incompetent the situation suggests that the respondent is $(M=10.35, t=4.71, p<0.0001, d=0.79)$; and how bad is the worst possible outcome is $(M=14.50, t=8.11, p<0.0001, d=1.35)$.

Posttreatment sample

Complete posttreatment data was collected from 25 of the participants, corresponding to 69.4 percent of the pretreatment sample. For practical reasons, the data were aggregated and analysed before 4 of the participants (11.1\% of the pretreatment sample) - for whom there was pretreatment data - had reached the posttreatment assessment point (i.e., they were still receiving active therapy at the time the data collection ceased). The reasons for participant attrition were not always clear as not all participants $(n=8)$ could be contacted regarding the posttreatment session. However, it was noted that one participant discontinued the study following a diagnosis of breast cancer during the study, one participant moved interstate, and one participant repeatedly did not attend their scheduled posttreatment assessment appointment.

There were no significant differences between the groups of participants with and without posttreatment data on any of the demographic variables, in terms of the presence of a medical condition, the use of psychotropic medication, engagement in therapy elsewhere, or in terms of the presence or absence of any given MINI-derived DSM-IV diagnosis. The presence of current alcohol abuse was an exception, with proportionately more individuals with compared to without alcohol abuse not attending the posttreatment session $(80.0 \%$ versus $22.5 \%$, respectively; $\chi^{2}=6.69, d f=1, p=0.01$ ). 
It is also noteworthy that the median number of sessions completed between the pre and posttreatment assessments was 9 sessions (Mean $=8.20 ; S D=3.08$; see Figure 1 ) and the median number of sessions that participants had attended in total by the posttreatment assessment was $10($ Mean $=10.12 ; S D=3.17)$. There were 3 participants from whom posttreatment data was collected within 4 sessions of the pretreatment session. This was either because improvement with treatment was rapid such that the participant did not feel the need to attend additional sessions ( $n=1)$ or because the participant concluded therapy for some other reason but was still able to attend the posttreatment assessment $(n=2)$. Each of these individuals was included in the analysis.

The mean duration of therapy between the pre and post-treatment assessments was 21.04 weeks $(S D=11.11$; Median $=17)$. The variability in treatment duration is consistent with the fact that therapy was based on individually tailored case formulation rather than a manualised approach, such that less frequent appointments were arranged with some participants towards the end of therapy to ensure that their progress persisted.

Following the administration of the posttreatment emotional reasoning items, participants were asked to what extent they believed that they remembered each of the emotional reasoning items from the pretreatment assessment (on a scale from $0=$ "Each item seemed completely new to me" to 100 = "I could remember almost every single situation extremely well"). The mean rating was $62.8(S D=23.54)$, suggesting at least some level of familiarity with each of the items.

Changes in symptom scores from pre to posttreatment

As could be expected, the symptom scores for anxiety and depression decreased significantly between the pre and posttreatment assessments (repeated measures $t$-test 95\% CI 
[4.60, 17.24] for BAI; repeated measures $t$-test 95\% CI [4.92, 13.64] for BDI-II; see Figure

2). The effect sizes for change on the BAI and BDI-II were 0.75 and 0.71 , respectively 4 .

Given the high rate of participant attrition, intent-to-treat analyses were also conducted. Again, the reductions in BAI and BDI-II scores were significant (repeated measures $t$-test 95\% CIs [2.96, 12.21] \& [3.14, 9.75] for BAI and BDI-II, respectively). Changes in emotional reasoning scores from pre to posttreatment.

The second hypothesis of this study was that emotional reasoning scores would decrease during a successful course of CBT for anxiety. Figure 3 summarises the patterns of scores for each of the respective emotional reasoning ratings at pre and posttreatment. Only one of the emotional reasoning scores changed to a significant extent during the course of therapy: ratings of incompetence decreased to a significant extent after controlling for multiple comparisons (pretreatment mean $=11.54[S D=13.22]$; posttreatment mean $=3.85$ $[S D=13.97]$; repeated measures $t$-test 95\% CI [2.13, 13.24]; see Figure 3). When an intent to treat analysis was conducted, the decrease in emotional reasoning incompetence ratings remained significant (repeated measures $t$-test 95\% CI [1.38, 9.30]).

Association between change in emotional reasoning for incompetence and changes in anxiety and depression.

The sample of participants completing treatment was small in size $(n=25)$. However, a post-hoc exploratory non-parametric correlation analysis ${ }^{5}$ was nonetheless conducted to determine whether decreases in emotional reasoning ratings for incompetence were associated with decreases in anxiety and depressive symptoms from pre to post therapy, as this bears upon an important question: Do changes in emotional reasoning scores occur in

\footnotetext{
${ }^{4}$ The effect size was calculated as: $\frac{\left(\bar{X}_{\text {Pretreatment }}-\bar{X}_{\text {Posttreatment }}\right)}{\text { SD of pretreatment scores }}$

${ }^{5}$ A non-parametric correlation analysis (Kendall's $\tau_{\mathrm{b}}$ ) was conducted given that the distribution of the overall population could not be assumed from the small sample of 25 participants from whom there was posttreatment data.
} 
concert with changes in anxiety and depressive symptoms? Change in emotional reasoning ratings of incompetence was positively correlated with change in BDI-II symptoms (Kendall's $\tau_{\mathrm{b}}=0.21$ ), although this correlation was not statistically significant in this small sample. The correlation between change in incompetence ratings and BAI scores was also 0.21 (Kendall's $\tau_{\mathrm{b}}$ ), although again, this was not statistically significant.

Association between pretreatment emotional reasoning scores and change in anxiety and depressive symptoms.

Given that high levels of emotional reasoning appear to be associated with elevated levels of anxiety symptoms (Arntz et al., 1995), it is plausible that high pretreatment levels of emotional reasoning may predict an attenuated degree of improvement in anxiety symptoms during a course of therapy. With this in mind, a further post-hoc correlation analysis was conducted to determine whether (high) levels of pretreatment emotional reasoning tendencies were associated with reduced improvements in anxiety and depressive symptoms during the course of therapy. Only pretreatment scores for how negative the situation was were associated with reduced degree of improvement in BAI symptoms (Kendall's $\tau_{\mathrm{b}}=-0.34, \mathrm{p}=$ 0.02): the other five emotional reasoning scores were not associated with the degree of improvement in BAI symptoms during the course of therapy.Discussion

This study aimed to determine whether a sample of individuals seeking treatment for an anxiety disorder demonstrated emotional reasoning tendencies. Consistent with the findings of Arntz et al. (1995), participants appeared to engage in emotional reasoning, as evidenced by emotional reasoning difference scores that were significantly greater than zero. In other words, averaged across the "objective” nature of scenario outcomes, participants tended to rate situations as more dangerous when an anxious emotional response was indicated. 
The present findings also extend those of Arntz et al. (1995) in that emotional reasoning tendencies were not only demonstrated for interpretations of how dangerous situations were, but also regarding how negative situations were perceived to be, as well as for ratings of how likely something bad was to occur, whether one would be able to cope, how incompetent the situation suggests that one is, as well as how bad the worst possible outcome would be. Thus, emotional reasoning was evident for both the perceived likelihood and cost of the situations as well as how incompetent one believes that one would be, this latter rating pertaining perhaps as much to depression as to anxiety per se. In other words, emotional reasoning appeared to be associated with a wide range of anxiety-related interpretations, not just the perceived danger of each given situation.

The second aim of the study was to determine whether emotional reasoning tendencies change during a routine course of individual CBT. This has not been investigated previously. In this respect, aside from emotional reasoning pertaining to one's perceived (in)competence, other emotional reasoning tendencies did not appear to change, even though participants significantly improved in anxiety and depressive symptoms. One can only speculate as to why incompetence-related emotional reasoning changed significantly when other emotional reasoning tendencies did not. Perhaps it is easier for individuals to learn to disentangle their emotional states at particular moments from (over)general interpretations of their competence than it is from interpretations about what might happen in the specific situation. However, if that was the case, it seems reasonable to also expect parallel reductions in ratings of being 'unable to cope', a similarly general interpretation.

Other emotional reasoning tendencies did not significantly change during the course of therapy. There are various explanations that could account for these findings. First, the apparent stability of emotional reasoning tendencies in both the present study and in previous prospective investigations of the construct (Berle \& Moulds, 2013a, 2013b) suggest that 
emotional reasoning might serve as a longstanding and potentially premorbid risk factor that increases an individual's risk of developing an anxiety disorder. Second the study serves only as a preliminary investigation and the sample size was too small to detect anything other than very large variations in emotional reasoning at both pre and posttreatment. Third, it is possible that emotional reasoning tendencies may take longer to change than the eight sessions (on average) that participants received between the pre and posttreatment assessments in the present study - or that emotional reasoning tendencies might change secondarily, once symptoms of anxiety and depression have already decreased. Fourth, it is possible that the scenarios-based procedure is insensitive to changes in emotional reasoning with treatment or that the posttreatment ratings were influenced by a practice/recall effect given that participants indicated at least some level of familiarity with each of the rated situations at posttreatment. Finally, the specific content of therapy sessions was not recorded, so it is not possible to determine to what extent emotional reasoning tendencies were recognised, discussed and addressed in therapy, and to what extent this may have varied between participants. On the one hand, the fact that therapists were using CBT-based approaches, and that the central place of emotional reasoning as a cognitive distortion in mainstream cognitive behavioural models of anxiety disorders, suggests that it would likely have been discussed at some point during therapy. On the other hand, participants received cognitive behavioural "treatment as usual" which did not include any prescribed content pertaining to emotional reasoning, so for many of the participants, the concept of emotional reasoning may have never been explicitly discussed or targeted in therapy at all. Preliminary evidence from a study where an experimental computerised task was used to modify the emotional reasoning tendencies of spider phobics (Lommen, Engelhard, van den Hout, Arntz, 2013) provides promise that specific strategies might be developed to address emotional reasoning in routine therapy settings as well. 
Conclusions from the present study need to be tempered by its limitations. The study lacked a control group. A non-clinical control group would have allowed direct comparison between the clinical and non-clinical participants to determine whether emotional reasoning is more prominent in individuals seeking treatment for an anxiety disorder compared with those in the population at large. However, the findings of Arntz et al. (1995) have already provided some support for this possibility. There may have been variations in how CBT was administered between therapists, and the small size of the sample precluded a statistical control of this. Also, many participants required more than 12 sessions of therapy, such that the "posttreatment” assessment may have occurred before additional gains were made. Perhaps emotional reasoning is a process that takes longer to change than other cognitive processes and biases. Finally, the sample comprised a heterogeneous group of individuals with anxiety disorders and it remains possible that there are differential changes in emotional reasoning between different disorder groups. For instance, emotional reasoning might change a lot during treatment for primary panic disorder, but relatively little for treatment of social anxiety disorder

As mentioned earlier, this was a preliminary investigation and additional studies using larger-size samples are required to adequately address the question of whether emotional reasoning changes with therapy. Nonetheless, these findings allow two tentative conclusions. First, individuals seeking treatment for an anxiety disorder appear to engage in emotional reasoning. Second, even though symptomatic improvement was evident in this small sample, a corresponding change in emotional reasoning tendencies did not appear to occur. 


\section{References}

American Psychiatric Association (2013). Diagnostic and Statistical Manual of Mental Disorders, fifth edition. Arlington, VA: American Psychiatric Association. doi: 10.1176/appi.books.9780890425596.807874

Arnau, R. C., Meagher, M. W., Norris, M. P., \& Bramson, R. (2001). Psychometric evaluation of the Beck Depression Inventory-II with primary care medical patients. Health Psychology, 20, 112-119. doi: 10.103W0278-6133.20.2.U2

Arntz, A., Rauner, M., \& van den Hout, M. (1995). "If I feel anxious, there must be danger": ex-consequentia reasoning in inferring danger in anxiety disorders. Behaviour Research and Therapy, 33, 917-925. doi: 10.1016/0005-7967(95)00032-S

Beck, A. T., \& Emery, G. (1985). Anxiety Disorders and Phobias: A Cognitive Perspective. New York: Basic Books.

Beck, A. T., Epstein, N., Brown, G., \& Steer, R. A. (1988). An inventory for measuring clinical anxiety: psychometric properties. Journal of Consulting and Clinical Psychology, 56, 893-897. doi: 10.1037/0022-006X.56.6.893

Beck, A. T., \& Steer, R. A. (1993). Beck Anxiety Inventory: Manual. San Antonio, TX: The Psychological Corporation, Harcourt Brace \& Company.

Beck, A. T., Steer, R. A., \& Brown, R. A. (1996). Beck Depression Inventory, 2nd Edition. San Antonio, TX: The Psychological Corporation.

Berle, D. \& Moulds, M. L. (2013a). Emotional reasoning processes and dysphoric mood: Cross-sectional and prospective relationships. PLOS ONE, 8(6), e67359. doi: 10.1371/journal.pone.0067359

Berle, D., \& Moulds, M. L. (2013b). An experimental investigation of emotional reasoning processes in depression. British Journal of Clinical Psychology, 52, 316-329. doi: 10.1111/bjc.12019 
de Beurs, E., Wilson, K. A., Chambless, D. L., Goldstein, A. J., \& Feske, U. (1997). Convergent and divergent validity of the Beck Anxiety Inventory for patients with panic disorder and agoraphobia. Depression and Anxiety, 6, 140-146. doi: 10.1002/(SICI)1520-6394(1997)6:4<140::AID-DA2>3.0.CO;2-G

Derogatis, L. R. (1983). SCL-90R administration, scoring, and procedures manual-II. Towson MD: Clinical Psychometric Research.

Engelhard, I. M., Macklin, M. L., McNally, R. J., van den Hout, M. A., \& Arntz, A. (2001). Emotion- and intrusion-based reasoning in Vietnam veterans with and without chronic posttraumatic stress disorder. Behaviour Research and Therapy, 39, 1339-1348. doi: 10.1016/S0005-7967(00)00101-7

Hicks, T. V., Leitenberg, H., Barlow, D. H., Gorman, . M., Shear, M. K., \& Woods, S. W. (2005). Physical, mental, and social catastrophic cognitions as prognostic factors in cognitive-behvaioral and pharmacological treatments for panic disorder. Journal of Consulting and Clinical Psychology, 73, 506-514. doi: 10.1037/0022-006X.73.3.506

Lommen, M. J. J., Engelhard, I. M., van den Hout, M. A., \& Arntz, A. (2013). Reducing emotional reasoning: An experimental manipulation in individuals with fear of spiders. Cognition and Emotion, 27, 1504-1512. doi: 10.1080/02699931.2013.795482

Muris, P., Merckelbach, H., \& Van Spauwen, I. (2003). The emotional reasoning heuristic in children. Behaviour Research and Therapy, 41, 261-272. doi: 10.1016/S00057967(02)00005-0

Sheehan, D. V., Lecrubier, Y., Sheehan, K. H., Amorim, P., Janavs, J., Weiller, E., . . . Dunbar, G. C. (1998). The Mini-International Neuropsychiatric Interview (M.I.N.I.): The development and validation of a structured diagnostic Psychiatric Interview for DSM-IV and ICD-10. Journal of Clinical Psychiatry, 59, 22-33. 
Steer, R. A., Ranieri, W. F., Beck, A. T., \& Clark, D. A. (1993). Further evidence for the validity of the Beck Anxiety Inventory with psychiatric outpatients. Journal of Anxiety Disorders, 7, 195-205. doi: 10.1016/0887-6185(93)90002-3

Uren, T. H., Szabó, M., \& Lovibond, P. F. (2004). Probability and cost estimates for social and physical outcomes in social phobia and panic disorder. Journal of Anxiety Disorders, 18, 481-498. doi: 10.1016/S0887-6185(03)00028-8

Verwoerd, J., de Jong, P. J., Wessel, I., \& van Hout, W. J. P. J. (2013). "If I feel disgusted, I must be getting ill": Emotional reasoning in the context of contamination fear. Behaviour Research and Therapy, 51, 122-127. doi: 10.1016/j.brat.2012.11.005 
Figure 1. Summary of participant numbers and attrition.

\section{Enrollment}

Assessed for eligibility $(n=48)$
Excluded $(n=11)$

Did not consent to participate:

- Due to childcare commitments $(n=3)$

- Due to lack of time $(n=4)$

- Reason unclear $(n=3)$

Other reasons:

- Due to high levels of distress at clinical assessment $(n=1)$

\section{Therapy}

\section{Posttreatment}

\section{Analysis}

- Mean number of sessions attended between pretreatment and posttreatment assessments $=8.20(S D=3.08$; Median = 9)

- Mean duration of therapy in weeks $=21.04(S D=11.11$; Median $=17$ )

- Mean number of sessions per participant that included discussion of cognitive therapy strategies ${ }^{\#}=4.32(S D=2.70$; Median $=4$ )

- Mean number of sessions per participant that included discussion of behavioural strategies ${ }^{\#}=2.80(S D=3.07$; Median = 2)
Lost to follow-up $(n=10)$

- Diagnosis of cancer $(n=1)$

- Moved interstate $(n=1)$

- Unclear $(n=8)$

Included in posttreatment assessment analyses despite posttreatment assessment occurring before the $12^{\text {th }}$ session $(n=4)^{\dagger}$

Excluded from pretreatment and posttreatment analysis due to computer software problem $(n=1)$

Analysed ( $n=36$ at pretreatment; $n=25$ at posttreatment)

* The majority of participants who completed therapy by the $12^{\text {th }}$ session had the frequency of their sessions reduced before the final session to ensure that progress was maintained.

\# A focus of a session was considered to involve at least 20 minutes of discussion. Thus, a single therapy session could have included more than one type of strategy (e.g., cognitive and behavioural).

"Due to a change of employment for the first author, four participants were assessed for their "posttreatment" assessment prematurely, before they had otherwise reached the posttreatment assessment time point. 
Table 1. Sample characteristics at pretreatment assessment $(N=36)$.

\begin{tabular}{lc}
\hline & Mean (Median; SD) \\
\hline Age & \multicolumn{1}{c}{ 32.6yrs (30; 12.1$)$} \\
\hline Gender & Female $=26(72.2 \%)$ \\
Marital status & Married or de facto $=18(50.0 \%)$ \\
Highest level of education completed & \\
$\quad$ Primary or high school & $14(38.8 \%)$ \\
$\quad$ Certificate, Diploma or Advanced & $17(47.2 \%)$ \\
$\quad$ Diploma & $5(13.8 \%)$ \\
Degree & $18(50.0 \%)$ \\
Currently engaged in paid employment & Any $=15(41.7 \%)$ \\
Current chronic medical condition & $7(19.4 \%)$ \\
$\quad$ Asthma & $11(30.6 \%)$ \\
Other & $22(61.1 \%)$ \\
Currently taking a psychotropic medication & $3(8.3 \%)$ \\
Currently receiving therapy elsewhere & \\
\# Some participants had multiple chronic medical conditions, including asthma. Thus, the \\
sum of individuals with asthma and with other medical conditions is greater than $n=15$.
\end{tabular}


Table 2. Numbers and percent of participants with co-occurring disorders at pretreatment ( $N$ $=36)$.

\begin{tabular}{lc}
\hline & $n(\%)$ \\
\hline Major depressive episode & $18(50.0 \%)$ \\
Dysthymia & $7(19.4 \%)$ \\
Panic disorder with or without & $7(19.4 \%)$ \\
Agorgaphobia & \\
Agoraphobia without Panic disorder & $4(11.1 \%)$ \\
Social anxiety disorder & $10(27.8 \%)$ \\
Obsessive-compulsive disorder & $4(11.1 \%)$ \\
Posttraumatic stress disorder & $5(13.9 \%)$ \\
Generalised anxiety disorder & $12(33.3 \%)$ \\
Specific phobia & $13(36.1 \%)$ \\
Hypochondriasis & $2(5.6 \%)$ \\
Alcohol abuse or dependence & $5(13.9 \%)$ \\
\hline
\end{tabular}


Figure 2. Changes in anxiety and depression self-report scores from pre to posttreatment $(n=$ 25).

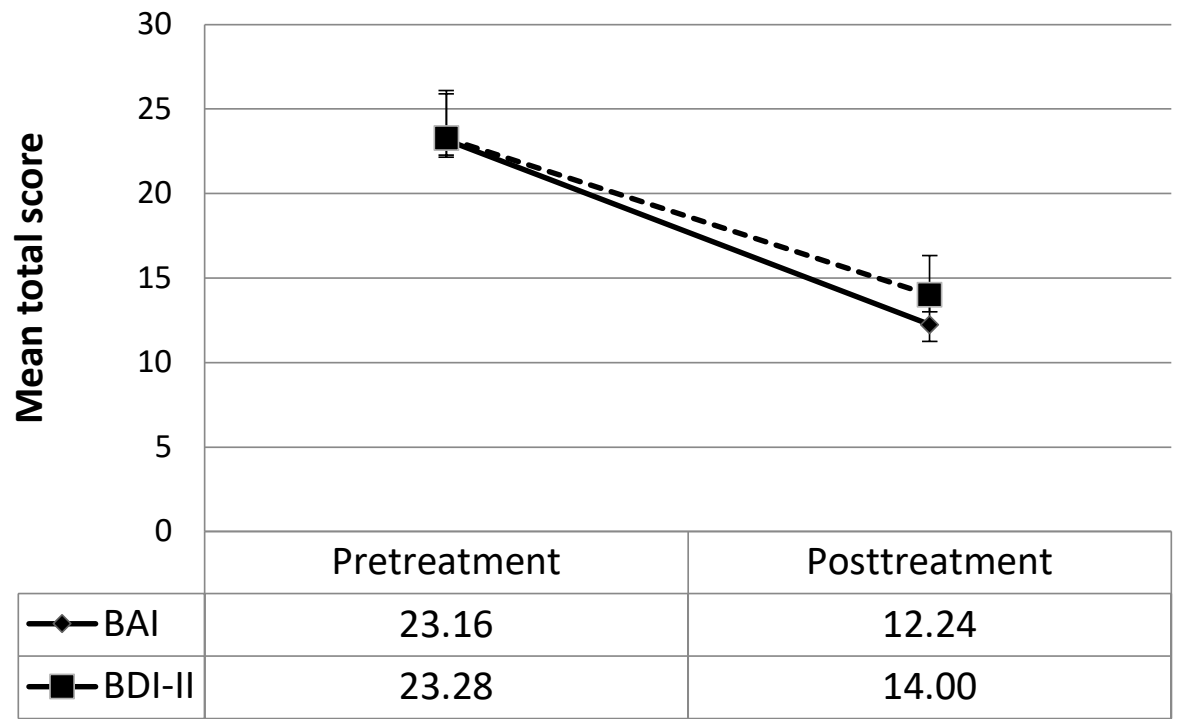

* Change in mean scores from pre to posttreament were significant (for BAI, 95\% CI [4.60, 17.24]; for BDI-II, 95\% CI [4.92, 13.64]).

Error bars indicate the Standard Error of the Mean. 
Figure 3. Mean emotional reasoning difference scores at pre and posttreatment assessment $(n=25)$.

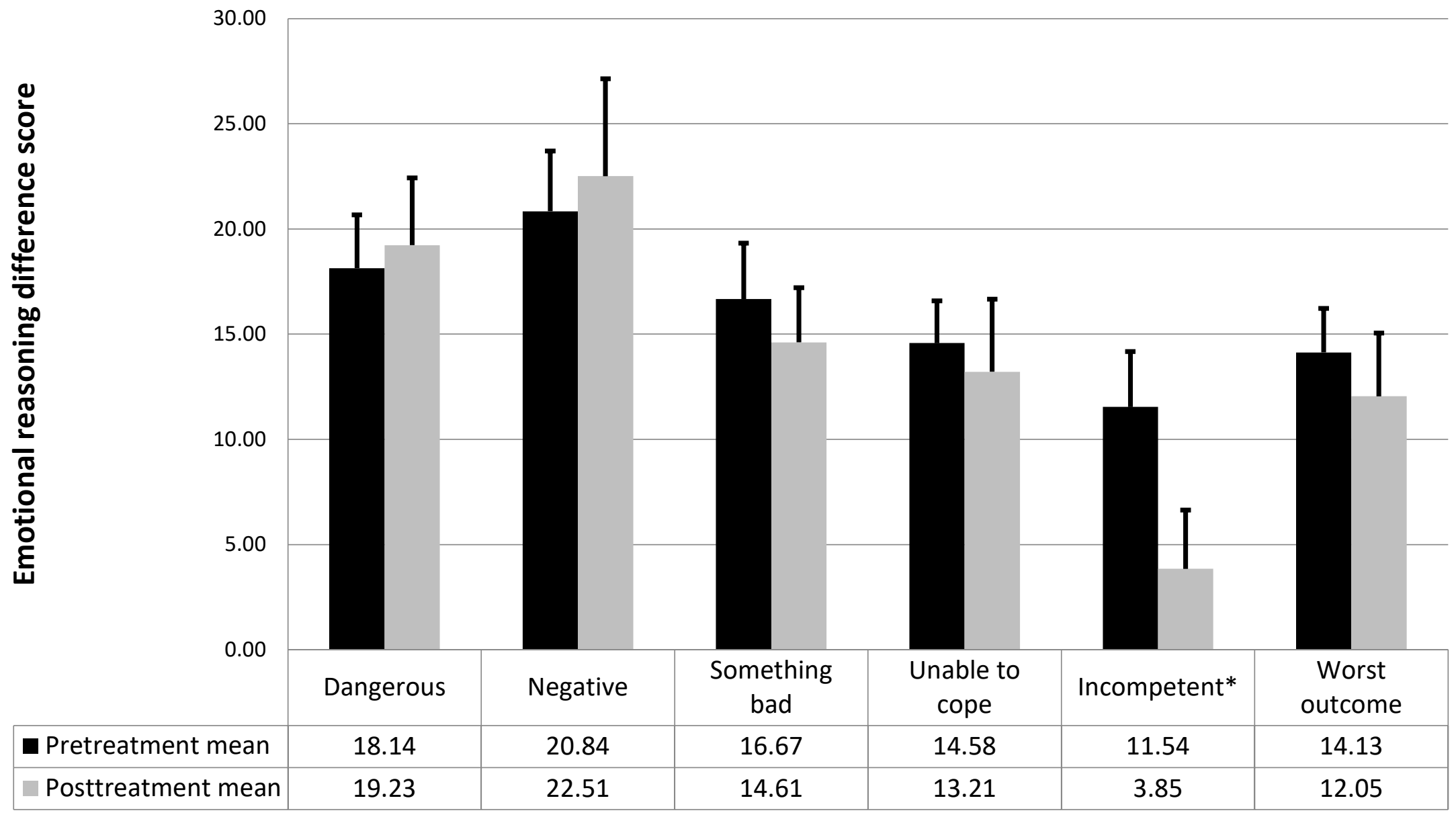

Error bars indicate the standard error of the mean.

* Ratings for "Incompetent" decreased to a significant extent from pre to posttreatment after correction for multiple comparisons (repeated measures $t$ 95\% CI [2.13, 13.24]). 